\title{
Analysis of radical cystectomy and urinary diversion complications with the Clavien classification system in an Italian real life cohort
}

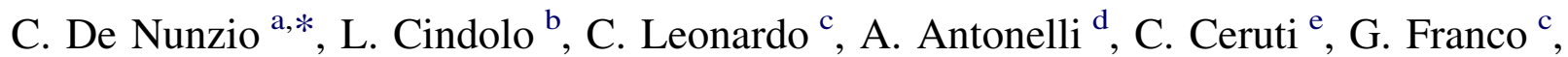
M. Falsaperla ${ }^{\text {h}}$, M. Gallucci ${ }^{n}$, M. Alvarez-Maestro ${ }^{i}$, A. Minervini ${ }^{j}$, V. Pagliarulo ${ }^{k}$, P. Parma ${ }^{f}$, S. Perdonà ${ }^{1}$, A. Porreca ${ }^{g}$, B. Rocco ${ }^{\mathrm{m}}$, L. Schips ${ }^{\mathrm{b}}$, S. Serni ${ }^{\mathrm{j}}$, M. Serrago ${ }^{\mathrm{m}}$, C. Simeone ${ }^{\mathrm{d}}$, G. Simone ${ }^{\mathrm{n}}$, R. Spadavecchia ${ }^{\mathrm{k}}$, A. Celia ${ }^{\mathrm{p}}$, P. Bove ${ }^{\mathrm{q}}$, S. Zaramella $^{\mathrm{o}}$, S. Crivellaro ${ }^{\mathrm{r}}$,

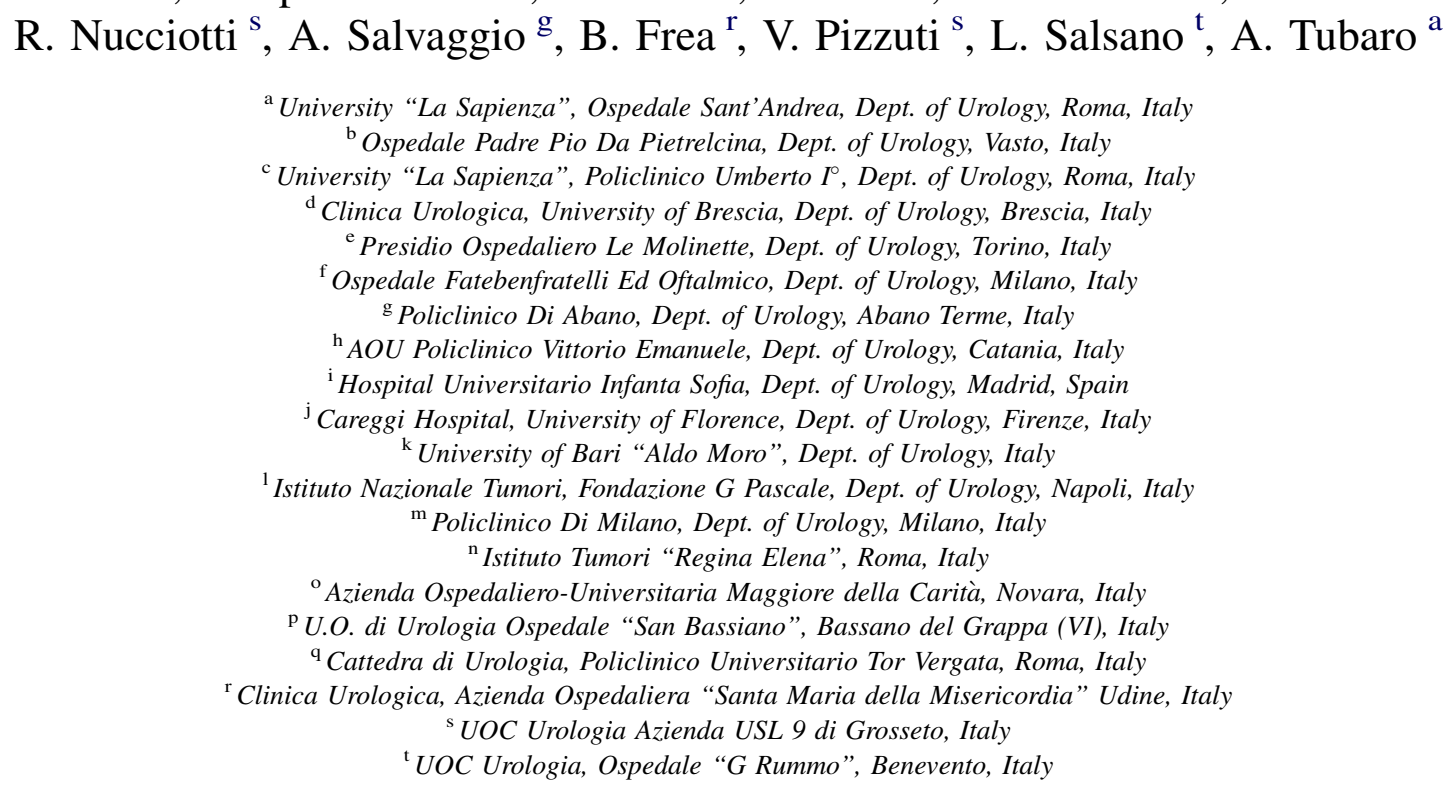

Accepted 13 March 2013

Available online 4 April 2013

\begin{abstract}
Introduction: Standardized methods of reporting complications after radical cystectomy (RC) and urinary diversions (UD) are necessary to evaluate the morbidity associated with this operation to evaluate the modified Clavien classification system (CCS) in grading perioperative complications of RC and UD in a real life cohort of patients with bladder cancer.

Materials and methods: A consecutive series of patients treated with RC and UD from April 2011 to March 2012 at 19 centers in Italy was evaluated. Complications were recorded according to the modified CCS. Results were presented as complication rates per grade. Univariate and binary logistic regression analysis were used for statistical analysis.

Results: Results and limitations: 467 patients were enrolled. Median age was 70 years (range 35-89). UD consisted in orthotopic neobladder in 112 patients, ileal conduit in 217 patients and cutaneous ureterostomy in 138 patients. 415 complications were observed in 302 patients and were classified as Clavien type I (109 patients) or II (220 patients); Clavien type IIIa (45 patients), IIIb (22 patients); IV (11 patients) and V (8 patients). Patients with cutaneous ureterostomy presented a lower rate (8\%) of CCS type $\geq$ IIIa $(p=0.03)$. A longer operative time was an independent risk factor of CCS $\geq$ III (OR: $1.005 ; \mathrm{CI}: 1.002-1.007$ per minute; $p=0.0001$ ).
\end{abstract}

\footnotetext{
* Corresponding author. Tel.: +39 0633777716; fax: +390633775428.

E-mail address: cosimodenunzio@virgilio.it (C. De Nunzio).
} 
Conclusions: In our study, RC is associated with a significant morbidity (65\%) and a reduced mortality (1.7\%) when compared to previous experiences. The modified CCS represents an easily applicable tool to classify the complications of RC and UD in a more objective and detailed way.

(C) 2013 Elsevier Ltd. All rights reserved.

Keywords: Bladder cancer; Cystectomy; Urinary diversion; Complications; Clavien

\section{Introduction}

Bladder cancer represents the 7 th most common cancer in men and the 17 th in women. ${ }^{1,2}$ Since the early 1960 s, radical cystectomy and pelvic lymphadenectomy have been considered the standard management of muscle invasive bladder cancer (MIBC) and a valid option for selected patients with high grade non muscle invasive bladder cancer (NMIBC). ${ }^{3-6}$ Notwithstanding improvements in surgical technique, technology and preoperative care have reduced the perioperative complication rate and even lowered the perioperative mortality rate from nearly $20 \%$ to less than $2 \%$; radical cystectomy (RC) is still associated with a significant perioperative and long term morbidity ranging from $19 \%$ to $64 \%$ according to the different series. ${ }^{7}$ However, complications associated with $\mathrm{RC}$ have been frequently classified as minor or major based on different definitions, which made an effective comparison of the different series impossible and highlight the fact that standardized methods for reporting data on surgical complications or morbidity after RC are urgently needed. ${ }^{7}$ The Clavien classification system (CCS) for surgical complications was originally developed in the 1990s and thereafter modified and validated ${ }^{6,7}$; nowadays it will probably represent the upcoming standard for both reporting complications and quality standard. ${ }^{8-10}$ Few retrospective studies, mostly in tertiary referral centers with a long enrollment period and focusing exclusively on ileal urinary diversions, examined complications using a CCS and have reported rates between $48 \%$ and $74 \% .^{11-13}$

The aim of our study was to prospectively evaluate, in a one-year multicenter Italian cohort of patients, the applicability of the modified CCS in grading perioperative complications of RC and urinary diversions (UDs) in patients with bladder urothelial carcinoma.

\section{Materials and methods}

\section{Patients and samples}

A consecutive series of patients treated with open RC, lymphadenectomy and UD from April 2011 to March 2012 at 19 centers in Italy were evaluated for complications occurring up to the end of the third postoperative month. The indications for surgery included muscle invasive urothelial carcinoma, selected patients with high grade NMIBC, BCG resistant carcinoma in situ and NMIBC that cannot be managed with transurethral resection. No exclusion criteria were considered. Age, sex, co-morbidities, smoking history, anthropometric parameters including body mass index (BMI), American Society of Anaesthesiologists (ASA) score, history of previous medical and surgical treatments and details of surgery were recorded from all patients. Clinical and pathological stages were evaluated for all the patients according to the TNM 2010 staging system. ${ }^{14}$ Antiaggregants and anticoagulant drugs were stopped at least 1 week before the operation. Daily administration of subcutaneous lowmolecular weight heparin was initiated $12 \mathrm{~h}$ before surgery and maintained for at least 4 weeks postoperatively. Polyethylene glycol solution was routinely administered the afternoon before surgery for bowel preparation. An elastic compressive stocking was used as mechanical prophylaxis for deep vein thrombosis (DVT) and third-generation cephalosporin was administered intravenously when anesthesia was initiated and maintained for 5 days postoperatively.

\section{Surgical technique}

All operations were performed by an expert qualified uro-oncology consultant in each center.

$\mathrm{RC}$ was performed according to the procedure suggested by the International Consultation on Bladder Cancer. ${ }^{15}$ Lymph node dissection extension included the removal of all lymphatic tissues around the common iliac, external iliac, internal iliac arteries and from the obturator region bilaterally and the presacral region. ${ }^{15,16}$ UD consisted of cutaneous ureterostomy according to the Glenn technique $^{17,18}$; ileal conduit according to the Bricker or Wallace technique $^{11,19}$ and ileal orthotopic neobladder according to the Studer or Camey or Padua techniques. ${ }^{16,19,20}$

All uretero-intestinal and uretero-cutaneous anastomosis were stented intraoperatively regardless of the form of UDs for at least 12 days. Patients with orthotopic neobladders underwent a contrast cystogram 3 weeks postoperatively and the urethral catheter was removed provided no significant leakage was observed.

\section{Complications evaluation}

All complications within 90 days of surgery were prospectively recorded and classified according to the modified 
$\mathrm{CCS}^{8}$ which stratifies perioperative complications into five grades and focuses mainly on the therapeutic consequences of a complication, emphasizing the level of intervention required to managed it. ${ }^{21}$ Likewise, perioperative mortality was defined as death from any cause within 90 days of surgery.

\section{Statistical analysis}

Statistical analysis was performed using the S-PSS 12.0 software (Chicago, USA). Differences between groups of patients in medians for quantitative variables and differences in distributions for categorical variables were tested with the Kruskal Wallis one way analysis of variance and chi-square test, respectively. Using multiple logistic regression with the enter method, the significant statistical variables as assessed in the univariate analysis were entered and investigated as predictors of severe complications (CCS $\geq$ III) vs. no or low-grade (CCS $\leq$ II) complications. The variables considered for entry into the model included age, sex (categorical), presence of hydronephrosis (categorical), ASA score (categorical variable), operative time, pathological stage (categorical) and type of urinary diversion (categorical). An alpha value of 5\% was considered as the threshold for significance. Data are presented as median (range). Odds ratios and 95\% CI's were calculated for the parameters in each group using no and low grade complications as a reference group.

\section{Results}

\section{Patient characteristics}

467 patients were prospectively and consecutively enrolled. Descriptive data of the cohort are summarized in Table 1.

None of the patients received neoadjuvant chemotherapy. All patients underwent RC and lymph nodes dissection. UD consisted in orthotopic neobladder in 113 patients, ileal conduit in 217 patients and bilateral cutaneous ureterostomy in 138 patients. Median operative time was 270 min (95-720); median estimated blood loss was 1290 cc (500-2000); median time to fluid intake was 5 days $(2-28)$. Median time to stool passage was 6 days (3-28). Median length of stay was 13 (6-119) days. Pathological stage was T0 in 28 patients; T1 in 36 patients (4 G1; 7G2 and $25 \mathrm{G} 3$ ); T2 in 103 patients (44 G2 and 59 G3); T3 in $165(35.5 \%)$ patients (55 G2 and $110 \mathrm{G} 3)$; T4 in 99 patients (38 G2 and $61 \mathrm{G} 3$ ) and CIS in 36 patients (7.8\%). Overall 141 patients presented a pathological $\mathrm{N}$ stage $\geq 1$.

\section{Clavien classification system evaluation}

A total of 415 complications were recorded in 302 patients with an incidence of perioperative complications of
Table 1

Descriptive preoperative data of 467 patients undergoing radical cystectomy.

\begin{tabular}{ll}
\hline Age (yrs) & $70(35-89)$ \\
Gender & Male: 379 patients \\
& Female: 88 patients \\
Smoker & 246 patients \\
Anticoagulant treatment & 140 patients \\
Diabetes mellitus & 88 patients \\
Hypertension & 278 patients \\
Ischemic heart disease & 93 patients \\
BMI $\left(\mathrm{kg} / \mathrm{m}^{2}\right)$ & $25(18-46)$ \\
Hemoglobin level $(\mathrm{g} / \mathrm{dl})$ & $13(9-17)$ \\
Chronic renal insufficiency $(\mathrm{GFR}<59 \mathrm{ml} / \mathrm{min})$ & 35 patients \\
Hydronephrosis $(\%)$ & 98 patients \\
ASA score & \\
I & 49 patients \\
II & 195 patients \\
III & 195 patients \\
IV & 28 patients \\
Tumor size (cm) & $4(1-12)$ \\
Tumor lesions (number\#) & $1(1-15)$ \\
\hline
\end{tabular}

*Data as median (range).

Table 2

Complications of radical cystectomy classified according to the modified Clavien system.

\begin{tabular}{lc}
\hline Clavien classification & Complications \\
\hline Grade $\mathbf{1}$ & $\mathbf{1 0 9}$ \\
Ileus paralytic & 25 \\
Infections & 31 \\
Transient elevation of creatinine & 16 \\
Metabolic imbalance & 10 \\
Transient reduction of Hb & 12 \\
Lymph discharge & 6 \\
Transitory ischemic attack & 3 \\
Dyspnea & 6 \\
Grade 2 & $\mathbf{2 2 0}$ \\
Blood transfusions & 182 \\
Infections & 20 \\
Small bowel obstruction & 17 \\
Deep venous trombosis & 1 \\
Grade 3a & $\mathbf{4 5}$ \\
Urinary leakage & 16 \\
Wound or pelvic Infections & 8 \\
Hydronephrosis & 5 \\
Small bowel obstruction & 8 \\
Lymphocele & 3 \\
Pneumotorax & 2 \\
Pelvic hematoma & 3 \\
Grade 3b & $\mathbf{2 2}$ \\
Urinary leakage & 6 \\
Wound infections & 6 \\
Small bowel obstruction or leakage & 9 \\
Pelvic hematoma & 1 \\
Grade 4a & $\mathbf{4 1 5}$ \\
Myocardial infarction & $\mathbf{9}$ \\
Lung failure & 4 \\
Kidney failure & 4 \\
Grade 4b & 1 \\
Urosepsis & $\mathbf{2}$ \\
Grade 5 & 2 \\
Overall & \\
&
\end{tabular}


Table 3

Analysis of the complications classified according to the CCS and perioperative patient's characteristics.

\begin{tabular}{|c|c|c|c|}
\hline & No complications & Complications & $p$ \\
\hline Patients & 165 & 302 & \\
\hline Age (yrs) & $71(65-76)$ & $70(35-89)$ & 0.588 \\
\hline Female & 23 & 64 & 0.054 \\
\hline BMI $\left(\mathrm{kg} / \mathrm{m}^{2}\right)$ & $25.5(23-34)$ & $25(22-46)$ & 0.616 \\
\hline Hemoglobin level (g/dl) & $13(9-17)$ & $12(9-16)$ & 0.254 \\
\hline Hydronephrosis & 28 & 70 & 0.07 \\
\hline Diabetes mellitus & 26 & 62 & 0.218 \\
\hline Hypertension & 98 & 180 & 0.521 \\
\hline Ischemic heart disease & 34 & 59 & 0.73 \\
\hline Chronic renal failure & 27 & 8 & 0.075 \\
\hline \multicolumn{4}{|l|}{ ASA score } \\
\hline I & 15 & 34 & \multirow[t]{4}{*}{0.232} \\
\hline II & 79 & 116 & \\
\hline III & 65 & 130 & \\
\hline IV & 6 & 22 & \\
\hline Tumor size $(\mathrm{cm})$ & $4(1-12)$ & $4(1-10)$ & 0.347 \\
\hline \multicolumn{4}{|c|}{2010 TNM pathological T stage } \\
\hline $\mathrm{T} 1$ or less & 17 & 47 & \multirow[t]{4}{*}{0.442} \\
\hline $\mathrm{T} 2$ & 45 & 58 & \\
\hline $\mathrm{T} 3-\mathrm{T} 4$ & 92 & 172 & \\
\hline CIS & 11 & 25 & \\
\hline 2010 TNM pathological N0 & 114 & 212 & 0.441 \\
\hline Operative time $(\min )$ & $256(95-640)$ & $272(95-720)$ & 0.189 \\
\hline Length of stay (days) & $12(6-40)$ & $14(6-119)$ & 0.534 \\
\hline
\end{tabular}

Data presented as median (range).

$65 \%$ (Table 2). Most complications were classified as Clavien type I (109 complications) or II (220 complications). Higher grade complications were observed: 45 Clavien type IIIa, 22 Clavien type IIIb, 11 Clavien type IV and 8
Clavien type V. Overall, 8 patients died and out of them three during the first postoperative month.

Overall, 182 patients required blood transfusion for perioperative bleeding. Most of the complications (311 complications) occurred during the initial hospitalization, and the remaining 104 following discharge. No significant association between clinical, pathological variables and the occurrence of any type of complications was observed (Table 3).

Patients with ureterocutaneostomy presented a higher rate of co-morbidity (hypertension, ischemic heart diseases, chronic renal failure), hydronephrosis and as a consequence a higher ASA score when compared to the ileal conduit or to the orthotopic neobladder (Table 4). Advanced disease (pT34) was also more frequent in patients who received a cutaneous ureterostomy (Table 4). Ileal conduit and orthotopic neobladder required a significantly longer operative time when compared to cutaneous ureterostomy $(p=0.001)$.

No significant differences $(p=0.991)$ were observed for the overall rate of complications in the three different UDs (112 complications in 138 patients for cutaneous ureterostomy; 201 complications in 217 patients for the ileal conduit and 102 complications in 112 patients for the orthotopic neobladder).

\section{High grade (CCS $\geq I I I a)$ complications evaluation}

Overall, 65 patients experienced high grade CCS ( $\geq$ IIIa) complications and 67 reinterventions (general or local anesthesia) were required: 14 for wound or pelvic infections; 22 for urinary anastomosis leakage; 17 for ileal perforation or

Table 4

Patient's characteristics according to the different type of urinary diversions.

\begin{tabular}{|c|c|c|c|c|}
\hline & Ureterocutanesostomy & Ileal conduit & Orthotopic neobladder & $p$ \\
\hline Patients & 138 & 217 & 113 & \\
\hline Age (yrs) & $75.5(35-89)$ & $71(47-86)$ & $63(36-77)$ & 0.001 \\
\hline BMI $\left(\mathrm{kg} / \mathrm{m}^{2}\right)$ & $24.6(23-28)$ & $26.4(22-46)$ & $25(27-40)$ & 0.237 \\
\hline Preoperative hemoglobin level (g/dl) & $11.6(9-13)$ & $12.7(9-17)$ & $13.2(10-17)$ & 0.008 \\
\hline Hydronephrosis $(\%)$ & 42 & 42 & 14 & 0.002 \\
\hline Diabetes mellitus & 29 & 42 & 17 & 0.485 \\
\hline Hypertension & 100 & 129 & 49 & 0.001 \\
\hline Ischemic heart disease & 40 & 41 & 12 & 0.001 \\
\hline Chronic renal failure & 20 & 12 & 3 & 0.001 \\
\hline ASA score & & & & 0.001 \\
\hline I & 9 & 23 & 17 & \\
\hline II & 46 & 89 & 60 & \\
\hline III & 67 & 95 & 33 & \\
\hline IV & 16 & 10 & 2 & \\
\hline Tumor size $(\mathrm{cm})$ & $5(1-10)$ & $4(1-12)$ & $4(1-7)$ & 0.191 \\
\hline 2010 TNM path. T stage & & & & 0.008 \\
\hline $\mathrm{T} 1$ or less & 14 & 34 & 16 & \\
\hline $\mathrm{T} 2$ & 32 & 39 & 32 & \\
\hline $\mathrm{T} 3-\mathrm{T} 4$ & 88 & 126 & 50 & \\
\hline CIS & 4 & 18 & 14 & \\
\hline 2010 TNM path. N0 & 84 & 152 & 80 & 0.064 \\
\hline Operative time (min) & $189(95-600)$ & $300(95-720)$ & $290(105-720)$ & 0.001 \\
\hline Estimated blood loss (cc) & $1340(500-2000)$ & $1600(500-1713)$ & $1140(1100 / 1365)$ & 0.644 \\
\hline Length of stay (days) & $12(6-119)$ & $13(6-60)$ & $14(6-35)$ & 0.036 \\
\hline
\end{tabular}

Data presented as median (range). 
Table 5

Patient's characteristics according to the different grade of complications.

\begin{tabular}{|c|c|c|c|}
\hline & $\begin{array}{l}\text { No complications or } \\
\mathrm{CCS} \leq \mathrm{II}\end{array}$ & $\mathrm{CCS} \geq \mathrm{IIIa}$ & $p$ \\
\hline Patients & 402 & 65 & \\
\hline Age (yrs) & $71(35-85)$ & $\begin{array}{l}70.3(10) ; 70 \\
(43-89)\end{array}$ & 0.279 \\
\hline Female/male ratio & 77 & 10 & 0.297 \\
\hline BMI $\left(\mathrm{kg} / \mathrm{m}^{2}\right)$ & $25(22-40)$ & $24(18-46)$ & 0.724 \\
\hline Hemoglobin level (g/dl) & $12.2(9-17)$ & $13(9-16)$ & 0.639 \\
\hline Hydronephrosis (\%) & 86 & 12 & 0.362 \\
\hline Diabetes mellitus & 70 & 18 & 0.06 \\
\hline Hypertension & 233 & 45 & 0.102 \\
\hline Ischemic heart disease & 80 & 13 & 0.550 \\
\hline Chronic renal failure & 27 & 8 & 0.096 \\
\hline ASA score & & & 0.259 \\
\hline I & 40 & 9 & \\
\hline II & 173 & 22 & \\
\hline III & 163 & 32 & \\
\hline IV & 26 & 2 & \\
\hline Tumor size $(\mathrm{cm})$ & $4(1-12)$ & $4.5(1-10)$ & 0.697 \\
\hline \multicolumn{3}{|c|}{$\begin{array}{l}2010 \text { TNM pathological T } \\
\text { stage }\end{array}$} & 0.761 \\
\hline $\mathrm{T} 1$ or less & 55 & 9 & \\
\hline $\mathrm{T} 2$ & 87 & 16 & \\
\hline $\mathrm{T} 3-\mathrm{T} 4$ & 227 & 37 & \\
\hline CIS & 33 & 3 & \\
\hline $\begin{array}{l}2010 \text { TNM pathological } \\
\text { N0 }\end{array}$ & 121 & 20 & 0.559 \\
\hline Operative time (min) & $260(95-640)$ & $343(108-720)$ & 0.001 \\
\hline Length of stay (days) & $12(6-50)$ & $20(6-119)$ & 0.001 \\
\hline
\end{tabular}

*Data presented as median (range).

occlusion; 5 for hydronephrosis; 4 for hematoma, 2 for pneumotorax and 3 for lymphocele. In patients with CCS $\geq$ IIIa a longer operative time and length of stay were observed when compared to patients with no or low grade $(\mathrm{CCS} \leq \mathrm{II})$ complications (Table 5). Patients who received a cutaneous ureterostomy presented a significantly ( $p=0.03$ ) lower rate of high grade CCS $\geq 3$ (11 complications in 138 patients) when compared to ileal conduit (35 complications in 217 patients) and orthotopic neobladder (19 complications in 112 patients). On multivariate analysis, a longer operative time was confirmed to be a significantly independent risk factor of high grade (CCS $\geq$ IIIa) complications (OR: 1.005; CI: 1.002-1.007 per minute; $p=0.0001)$, despite the type of UD.

\section{Discussion}

\section{Radical cystectomy morbidity and mortality rate}

Radical cystectomy and pelvic lymphadenectomy is the standard treatment for patients with muscle invasive or high risk NMIBC although it is associated with a high risk of severe complications. ${ }^{11,22}$ In our prospective multicenter group of patients enrolled for one year and treated with RC and three different types of UD, we observed a low one month (0.63\%) and three months $(1.7 \%)$ mortality rate associated with an elevated (65\%) morbidity which was higher when compared to the data reported by Novara et al. ${ }^{11}$ and by Shabsigh et al. ${ }^{12}$ However, our group of patients were older (median 70 years vs. 65 and 68 respectively) and with a higher prevalence of comorbidities as diabetes or arterial hypertension. Furthermore, they ${ }^{11,12}$ retrospectively evaluated only patients treated in a single tertiary referral, enrolled over a long range of time (5-10 years) using exclusively an ileal UD (ileal conduit, continent pouch or orthotopic neobladder). ${ }^{11,12}$ Our experience included patients from 19 different teaching and non-teaching hospitals and better reflects the real life activity in Italy. In particular, cutaneous ureterostomy was the selected UD in about $25 \%$ of our patients. Although cutaneous ureterostomy is performed worldwide in about $2 / 10 \%$ of patients treated with $\mathrm{RC}^{23}$ it is still a common procedure in Italy and in a real life setting it could be indicated in patients with advanced bladder cancer and higher co-morbidities as observed in our series. In this group of patients we particularly observed, together with advanced bladder cancer, a higher incidence of hydronephrosis and co-morbidities when compared to patients receiving an ileal UD (Table 4). Most of these procedures were performed without any curative intent or to improve patients' survival but only as a palliative procedure to avoid future worsening of the patients' quality of life that may derive from hematuria with clot formation, urinary retention, pain from infiltration of the pelvic nerves and renal failure.

The most common complication categories were hematological (198 complications), gastrointestinal (57 complications), infectious (51 complications), wound related (16 complications) and genito-urinary (30 complications). Although 415 complications were observed in 302 patients, most of them (329 complications) were considered not severe $(\mathrm{CCS} \leq \mathrm{II})$ according to the $\mathrm{CCS}$. In this group of patients the most common complication was blood transfusion which was necessary in 182 patients during the perioperative observation time with an average estimated blood loss of $1200 \mathrm{ml}$ which is in the same range reported by other groups $(560-3000 \mathrm{ml}) .^{24,25}$ The non significant differences in terms of estimated blood loss were observed among the three different UDs which underline how this type of complication can be attributable to the $\mathrm{RC}$ procedure independently from the UD procedure.

\section{Radical cystectomy and high grade (CCS $\geq I I I a)$ complications}

In our analysis, a small number of patients (65 patients) experienced high grade complications $(\mathrm{CCS} \geq 3 \mathrm{a})$ and most of them were related to small bowel obstruction (17 complications) or anastomosis urinary leakage and hydronephrosis (27 complications). We confirmed in our series how CCS $\geq$ IIIa complications are mostly related to the UD procedure and particularly to the ileal resection and 
anastomosis or to the ureteric and urethra anastomosis. The relation of CCS $\geq$ IIIa complications with ileal UD can also explain the similar trend of the high rate of these complications observed in all the studies on robotic, open or laparoscopic RC where the ileal UD is always performed through a midline incision ${ }^{11,21,25,26}$ independently from the open, laparoscopic or robotic approach.

Furthermore, this hypothesis is confirmed in our series by the group of patients with cutaneous ureterostomy where we observed a lower rate ( $8 \%$ ) of CCS $\geq$ IIIa complications when compared to the ileal conduit (16\%) and the ileal orthotopic neobladder (17\%). Although we acknowledge that ileal conduit and orthotopic neobladder should be considered as the standard UD in patients treated with $\mathrm{RC}$, in our experience, in patients with significant co-morbidities, cutaneous ureterostomy could be proposed and discussed in selected patients in order to reduce the morbidity associated with ileal UD.

Multivariate analysis showed that operative time was a statistically significant independent predictor of CCS $\geq$ IIIIa despite the type of UD. Although our results could be in relation to the reduced statistical power associated with the smaller number of patients who experienced CCS $\geq$ IIIa complications (65 patients), it could reflect that a longer operative time is required in patients with technically demanding procedures in relation to the patient's habit, tumor stage, previous surgery, or intraoperative complications; it can also reflect particular anesthesiological needs and can induce metabolic imbalance, resulting in a higher rate of high grade postoperative complications. The average length of stay after a $\mathrm{RC}$ ranges between 7 and 34 days according to the different centers, with significant differences between Europe (longer length of stay) and American (shorter length of stay) hospitals. ${ }^{11,12}$ In our experience the median length of stay was 13 days (range 6-119) and it was significantly longer in patients with high grade complications. However, compared to other series our median length of stay was shorter probably in relation to the different types of UDs included in our series. In particular, patients with cutaneous ureterostomy presented significant shorter hospitals stay when compared to ileal UDs.

\section{Study limitations}

Despite the fact that to our knowledge this study represents the largest multi-institution analysis of RC and UDs using the CCS, we must acknowledge some limitations. The relationship between hospital volume and RC outcome in terms of morbidity and mortality is well established. ${ }^{21} \mathrm{In}$ our series 19 different centers from teaching and non-teaching hospitals were involved in this project and the results obtained in our experience could not be referred or transferable to high volume cancer centers. Even though some authors ${ }^{21}$ took into consideration 50 procedures per year as the desirable cystectomy rate in order to minimize the possible complications, the International Consultation on Bladder cancer has recently proposed an annual rate of $10 \mathrm{RC}$ procedures per hospital as an adequate rate. ${ }^{15} \mathrm{In}$ our series, all the centers involved reached this standard (median $21 \mathrm{RC}$ per center, range 13-40). Another possible limitation of our study is related to the small sample size when compared to other larger American series. ${ }^{12,21}$ However, we decided to reduce the enrollment period to one year and consequently the sample size in order to have a precise and real life picture of these procedures and to minimize the possible influence of the surgical and technological progress which has been particularly evident year after year in the last decades. Finally, although ileal conduit and orthotopic neobladder are considered the standard $\mathrm{UDs}^{23}$ in our series, about $25 \%$ of the patients received a cutaneous ureterostomy which influenced our results and reduced the possibility of comparing our entire group of patients with the available evidence. However, we present a real life experience in our study where this type of UD is still a frequent option. Furthermore, including the patients in our series, we better highlighted and demonstrated the direct relationship between high grade complications and ileal UD.

\section{Conclusions}

In our study we confirmed, in one year of a real life cohort of patients, that the CCS is a feasible method to evaluate early morbidity after RC and UDs. Although most of the complications were minor, $12 \%$ of the patients experienced high grade complications and most of them were observed in patients who received ileal UDs (orthotopic neobladder or ileal conduit). Although mortality rate has dropped significantly over the last decades, perioperative complications remain common.

\section{Acknowledgment statement}

The authors acknowledge Kimberlee Manzi from the University "La Tuscia", Viterbo, Italy, who provided English editing support.

\section{Conflict of interest statement}

All authors declare no financial or personal relationships with other people or organizations that could inappropriately influence (bias) their work.

\section{References}

1. Colombel M, Soloway M, Akaza H, et al. Epidemiology, staging, grading, and risk stratification of bladder cancer. Eur Urol Suppl 2008;7(10):618-26. 
2. Kirkali Z, Chan T, Manoharan M, et al. Bladder cancer: epidemiology, staging and grading, and diagnosis. Urology 2005;66(6 Suppl. 1):4-34.

3. Babjuk M, Oosterlinck W, Sylvester R, Kaasinen E, Bohle A, PalouRedorta J. EAU guidelines on non-muscle-invasive urothelial carcinoma of the bladder. Eur Urol 2008;54(2):303-14.

4. van Rhijn BW, Burger M, Lotan Y, et al. Recurrence and progression of disease in non-muscle-invasive bladder cancer: from epidemiology to treatment strategy. Eur Urol 2009;56(3):430-42.

5. De Nunzio C, Carbone A, Albisinni S, et al. Long term experience with early single mitomycin $\mathrm{C}$ instillations in patients with low-risk nonmuscle-invasive bladder cancer: prospective single centre randomized trial. World J Urol 2011 Aug;29(4):517-21.

6. Gschwend J, Retz M, Kuebler H, Autenrieth. Indications and oncological outcome of radical cystectomy for urothelial bladder cancer. Eur Urol Suppl 2010;9:10-8.

7. Liedberg F. Early complications and morbidity of radical cystectomy. Eur Urol Suppl 2010;9:25-30.

8. Dindo D, Demartines N, Clavien PA. Classification of surgical complications: a new proposal with evaluation in a cohort of 6336 patients and results of a survey. Ann Surg 2004;240:205-13.

9. Clavien PA, Sanabria JR, Strasberg SM. Proposed classification of complications of surgery with examples of utility in cholecystectomy. Surgery 1992;111:518-26.

10. Tefekli A, Ali Karadag M, Tepeler K, et al. Classification of percutaneous nephrolithotomy complications using the modified Clavien grading system: looking for a standard. Eur Urol 2008;53(1):184-90.

11. Novara G, De Marco V, Aragona M, et al. Complications and mortality after radical cystectomy for bladder transitional cell cancer. J Urol 2009;182:914-21.

12. Shabsigh A, Korets R, Vora KC, et al. Defining early morbidity of radical cystectomy for patients with bladder cancer using a standardized reporting methodology. Eur Urol 2009;55:164-76.

13. Bostrom PJ, Kossi J, Laato M, Nurmi M. Risk factors for mortality and morbidity related to radical cystectomy. BJU Int 2009;103:191-6.

14. Sobin LH, Gospodarowicz MK, Wittekind C. International union against cancer. TNM classification of malignant tumours. 7th ed. Hoboken, NJ: Wiley-Blackwell; 2010.
15. Gakis G, Efstathiou J, Lerner SP, et al. ICUD-EAU International Consultation on Bladder Cancer 2012: radical cystectomy and bladder preservation for muscle-invasive urothelial carcinoma of the bladder. Eur Urol 2012; http://dx.doi.org/10.1016/j.eururo.2012.08.009.

16. Tubaro Andrea, Santini Daniele, De Nunzio Cosimo, Zoccoli Alice, Iuliano Michele. Bladder cancer. In: Bologna M, editor. Biotargets of cancer in current clinical practice. New York: Springer Science Buisiness Media; 2012, p. 325-54.

17. De Nunzio C, Cicione A, Leonardo C, et al. Extraperiotoneal radical cystectomy and ureterocutaneostomy in octogerians. Int Urol Nephrol 2011;43(3):663-7.

18. Glenn JF, Alyea EP. Ureterocutaneous anastomosis. I. Experimental use of a surgical splint to prevent stricture. Trans Southeast Sect Am Urol Assoc 1960;1959:36-9.

19. Dahl DM, McDougal WS. Use of intestinal segments in urinary diversion. In: Wein AJ, Kavoussi LR, Novick AC, et al, editorss. Campbell-Walsh urology. 9th ed. Philadelphia: WB Saunders Co; 2006 , p. 2534-78. sect 15 , chapt 6 .

20. Pagano F, Artibani W, Ligato P, et al. Vescica ileale Padovana: a technique for total bladder replacement. Eur Urol 1990;17:49-54.

21. Hautmann R, de Petriconi R, Volkmer B. Lessons learned from 1,000 neobladders: the 90-day complication rate. J Urol 2010;184:990-4.

22. Lawrentschuk N, Colombo R, Hakenberg OW, et al. Prevention and management of complications following radical cystectomy for bladder cancer. Eur Urol 2010;57:983-1001.

23. Hautmann RE, Abol-Enein H, Davidsson T, et al. ICUD-EAU International Consultation on Bladder Cancer 2012: urinary diversion. Eur Urol 2012; http://dx.doi.org/10.1016/j.eururo.2012.08.050.

24. Novotny V, Hakenberg OW, Wiessner D, et al. Perioperative complications of radical cystectomy in a contemporary series. Eur Urol 2007;51:397-402.

25. Yuh BE, Nazmy M, Ruel NH, et al. Standardized analysis of frequency and severity of complications after robot-assisted radical cystectomy. Eur Urol 2012 Nov;62(5):806-13.

26. Khan MS, Elhage O, Challacombe B, Rimington P, Murphy D, Dasgupta P. Analysis of early complications of robotic-assisted radical cystectomy using a standardized reporting system. Urology 2011;77: 357-62. 